\title{
Immune checkpoint inhibitor associated reactivation of primary membranous nephropathy responsive to rituximab
}

\author{
Jamie S Lin (D) , ${ }^{1}$ Daniel Y Wang, ${ }^{2}$ Omar Mamlouk (D) , ${ }^{1}$ William F Glass, ${ }^{3}$ \\ Maen Abdelrahim, ${ }^{4}$ Cassian Yee, ${ }^{5,6}$ Ala Abudayyeh ${ }^{1}$
}

To cite: Lin JS, Wang DY, Mamlouk 0, et al. Immune checkpoint inhibitor associated reactivation of primary membranous nephropathy responsive to rituximab. Journal for ImmunoTherapy of Cancer 2020;8:e001287. doi:10.1136/ jitc-2020-001287

Accepted 27 August 2020

Check for updates

(C) Author(s) (or their employer(s)) 2020. Re-use permitted under CC BY-NC. No commercial re-use. See rights and permissions. Published by BMJ.

${ }^{1}$ Section of Nephrology, Division of Internal Medicine, University of Texas MD Anderson Cancer Center, Houston, Texas, USA

${ }^{2}$ Section of HematologyOncology, Department of Internal Medicine, Baylor College of Medicine, Houston, Texas, USA ${ }^{3}$ Department of Pathology and Laboratory Medicine, University of Texas Health Science Center McGovern Medical School, Houston, Texas, USA

${ }^{4}$ Department of Medical Oncology, Institute of Academic Medicine and Weill Cornell Medical College, Houston Methodist Cancer Center, Houston, Texas, USA

${ }^{5}$ Department of Melanoma Medical Oncology, Division of Cancer Medicine, University of Texas MD Anderson Cancer Center, Houston, Texas, USA

${ }^{6}$ Department of Immunology, University of Texas MD Anderson Cancer Center, Houston, TX, USA

Correspondence to

Dr Ala Abudayyeh;

aabudayyeh@mdanderson.org

\section{ABSTRACT}

The same mechanisms that mediate antitumor immunity from checkpoint inhibitors (CPIs) can also lead to unintended targeting of normal tissues, characterized as immune-related adverse events (irAEs). Those with pre-existing autoimmune disease are believed to be particularly vulnerable for exacerbating underlying autoimmunity or inducing severe irAEs. We report the first case of $\mathrm{CPI}$-associated reactivation of primary membranous nephropathy (MN) in a patient with pleural mesothelioma responding to immunotherapy. Due to its specificity in targeting B-lymphocytes, rituximab was used to treat primary MN with the expectation that this would not interfere with the benefits gained from T cell-mediated antitumor immunity. Rituximab was effective in treating $\mathrm{CPI}$-associated reactivation of $\mathrm{MN}$, and the patient was successfully rechallenged with nivolumab and maintained stable kidney function and sustained clinical antitumor effect. While exacerbation of pre-existing autoimmune diseases from CPIs is common, therapy for autoimmune reactivation can be rationally directed by an understanding of the immunosuppressive mechanism with goals of cancer treatment.

\section{BACKGROUND}

Immune checkpoint therapy has dramatically altered the treatment landscape of patients with cancer. However, the same mechanisms that mediate antitumor immunity can also lead to unintended targeting of normal tissues, characterized as immunerelated adverse events (irAEs). Those with pre-existing autoimmune disease are believed to be particularly vulnerable for exacerbating underlying autoimmunity or inducing severe irAEs. Primary membranous nephropathy (MN) is a common cause of nephrotic syndrome in adults. It is an antibody-mediated autoimmune glomerular disease, where approximately $70 \%$ of patients will produce autoantibodies to the M-type phospholipase A2 receptor (PLA2R) on glomerular podocytes. ${ }^{1}$ Fortunately, only approximately $30 \%$ of untreated patients with MN will progress to end-stage kidney failure. Immunosuppressive treatment is recommended for those at risk for progressive disease. MN can also be associated with malignancies, infections, and certain medications including checkpoint inhibitors (CPIs). ${ }^{2}$ The strong association between malignancy and $\mathrm{MN}^{34}$ can make it difficult to distinguish from CPI-associated MN. Furthermore, treatment of paraneoplastic glomerular diseases is achieved with treatment of the underlying cancer. Here we present a patient with history of primary $\mathrm{MN}$ ( $>10$ years) and malignant pleural mesothelioma. We report the first case of immune checkpoint inhibitor (CPI)-associated reactivation of primary MN responsive to rituximab in a patient with pleural mesothelioma on immune checkpoint therapy with durable complete remission.

\section{CASE PRESENTATION}

A 60-year-old man with clinical stage IA malignant pleural mesothelioma receiving off-label nivolumab, anti-programmed cell death-1 (PD-1) therapy, was referred to the University of Texas MD Anderson Cancer Center (MDACC) nephrology clinic for concern of reactivation of primary $\mathrm{MN}$.

The patient has had a history of primary MN for approximately 14 years. He was found to have nephrotic range proteinuria (3-4g/ day) on an annual physical examination and referred to a local nephrologist. Per the patient, initial workup was unrevealing and his proteinuria spontaneously improved to $<1.0 \mathrm{~g} /$ day. In 2011, he had recurrence of proteinuria and underwent kidney biopsy. At the time, PLA2R testing was unavailable; however, his biopsy findings in 2011 were suggestive of primary MN including thickened glomerular capillaries, glomerular basement membrane (GBM) pits and spikes, subepithelial intramembranous deposits (figure 1A-C), and absence of subendothelial and mesangial deposits. Screening for 


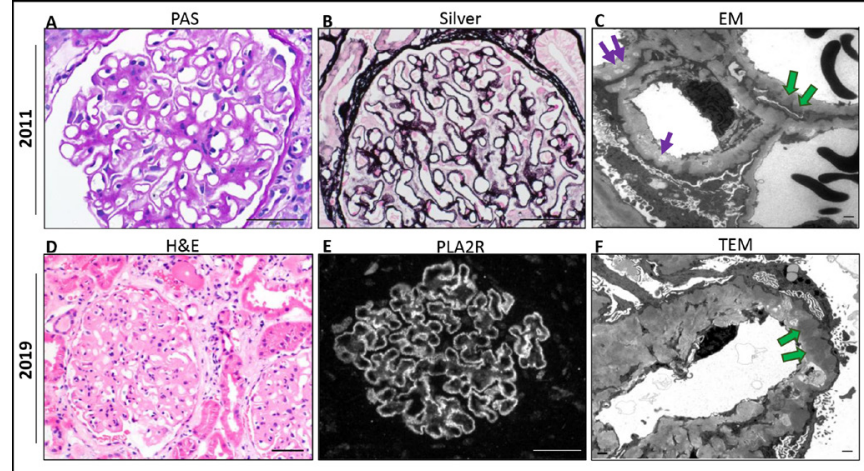

Figure 1 Renal biopsy 2011. (A) Periodic acid-Schiff (PAS) stain of glomerulus with thick capillary walls. (B) Jones methenamine silver (silver): basement membrane pits and spikes. (C) Electronic micrograph (EM): thickened glomerular basement membrane (GBM) 1000 nm, subepithelial, intramembranous deposits (green arrows) and electron-lucent intramembranous spaces (purple arrows). Renal biopsy 2019. (D) Hematoxylinand eosin (H\&E) stain thick glomerular capillaries with patchy mild to focally moderate infiltration by lymphocytes. (E) Phospholipase A2 receptor (PLA2R) stain is diffuse and granular with capillary distribution. (F) EM thickened GBM measuring $>3000 \mathrm{~nm}$ (normal 230-430 nm). Electron-dense subepithelial deposits (green arrow). Diffuse podocyte effacement. Scale bar: $50 \mu \mathrm{m}(\mathrm{A}-\mathrm{E})$ and $1 \mu \mathrm{m}(\mathrm{C}, \mathrm{F})$.

secondary causes of $\mathrm{MN}$ including: hepatitis B and $\mathrm{C}$ serologies, anti-nuclear antibody (ANA) titer, prostate-specific antigen (PSA) level, chest x-ray, and colonoscopy were unrevealing. Non-immunosuppressive therapy, including lisinopril and simvastatin, was initiated. While he was ultimately unable to tolerate lisinopril due to hyperkalemia, he went into spontaneous remission. Since 2011, he has only had one additional relapse in 2017 that also spontaneously remitted without use of steroids or immunosuppressive treatment.

In September 2018, he was diagnosed with malignant pleural mesothelioma (MPM) of the right hemithorax, biphasic with predominant sarcomatoid type. He was deemed not to be a candidate for surgery or combination chemotherapy due to poor performance status. Benefits of single-agent chemotherapy versus off-label use of nivolumab were discussed. After careful consideration, he proceeded with off-label nivolumab infusions $(240 \mathrm{mg}$ intravenous every 2 weeks), along with careful monitoring of his kidney function by his local nephrologist. Interval improvement in his cancer was seen on positron emission tomography/computed tomography (PET/CT) imaging. Shortly after starting nivolumab, his proteinuria began to increase. Additionally, he developed diabetic ketoacidosis and checkpoint inhibitor-induced type 1 diabetes with positive glutamic acid decarboxylase 65 (GAD65) antibody within 1 month of therapy (figure 2 ).

In November 2019, the patient came to nephrology clinic at MDACC for a second opinion. Notable labs included a serum albumin of $1.9 \mathrm{~g} / \mathrm{dL}$, an elevated serum creatinine (Cr) $1.25 \mathrm{mg} / \mathrm{dL}$ (baseline Cr $0.82-0.91 \mathrm{mg} /$ $\mathrm{dL}$ ), urinalysis with trace glucose 150 ; protein $>=500$,

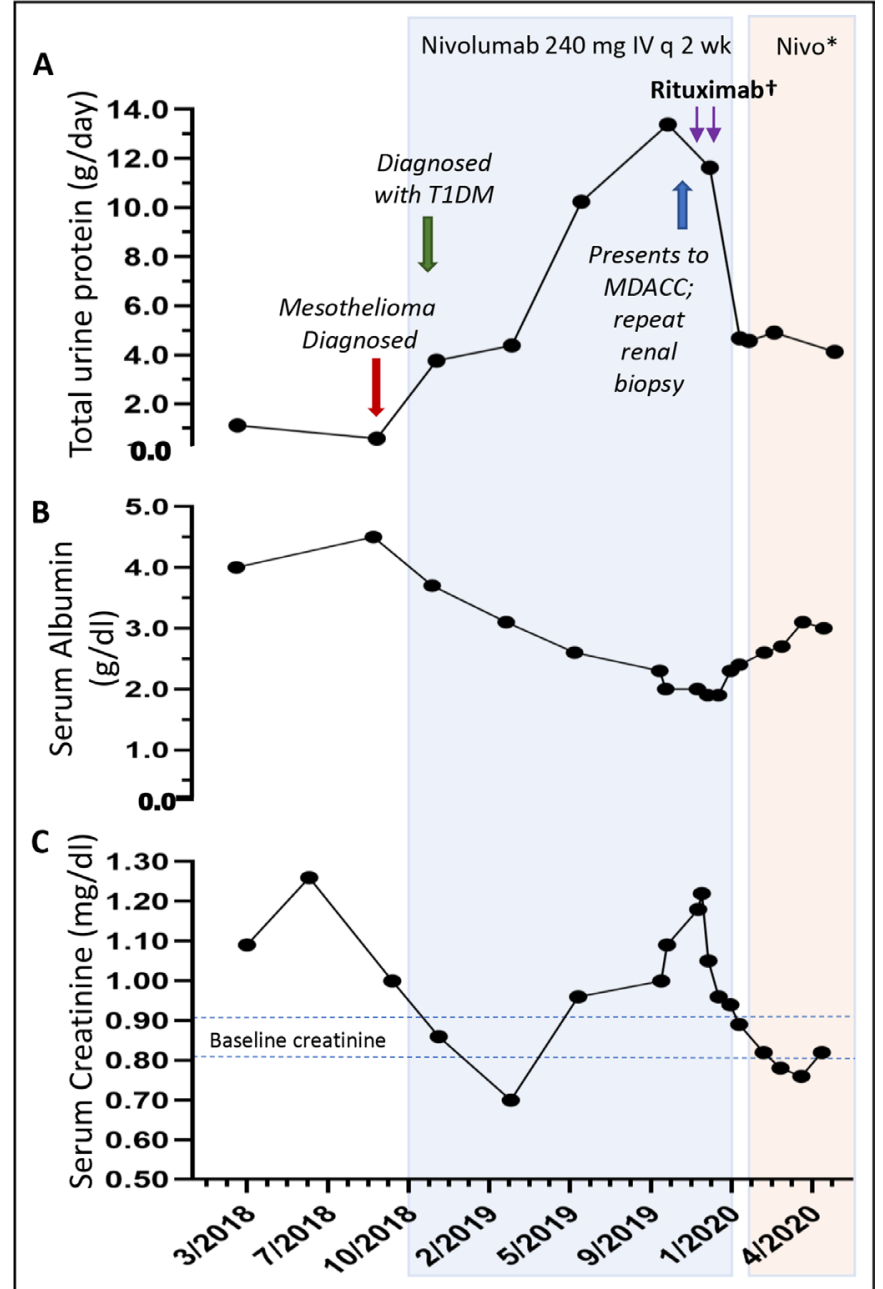

Figure 2 Timeline of events: (A) 24-hour urine protein was $<1 \mathrm{~g} / 24$ hours prior to during time of diagnosis and prior to nivolumab therapy. In November 2019, proteinuria peaked at $13.4 \mathrm{~g} / 24$ hours. (B) Serum albumin level nadired at $1.9 \mathrm{~g} /$ dL. (C) Serum creatinine peaked at $1.26 \mathrm{mg} / \mathrm{dL}$. *Nivolumab $480 \mathrm{mg}$ intravenous every month. †Rituximab $1 \mathrm{~g}$ intravenous administered on days 1 and 15. MDACC, University of Texas MD Anderson Cancer Center (MDACC); T1DM, type 1 diabetes mellitus.

negative bilirubin, ketones, blood, urobilinogen, nitrate, leucocyte esterase; white blood cells/high power field (HPF) : 1; red blood cells/HPF : 2; hyaline casts: 4 (high), and a 24-hour urine protein $13.4 \mathrm{~g} /$ day which had previously been $<1.0 \mathrm{~g} /$ day prior to therapy with nivolumab (figure 2). Hepatitis serologies and review of secondary causes of MN were again unremarkable.

Due to concern for reactivation of primary $\mathrm{MN}$ versus other CPI-nephritis etiologies, he underwent a kidney biopsy. Biopsy results suggested chronic reactivated primary MN. Light microscopy revealed thick glomerular capillaries (figure 1D) and immunofluorescence demonstrated capillary granular IgG and C3 deposits (not shown) and strongly positive PLA2R stain (figure 1E). Electron microscopy showed electron-lucent intramembranous spaces, subepithelial electron dense deposits, thickened GBM measuring $>3000 \mathrm{~nm}$ (average adult 


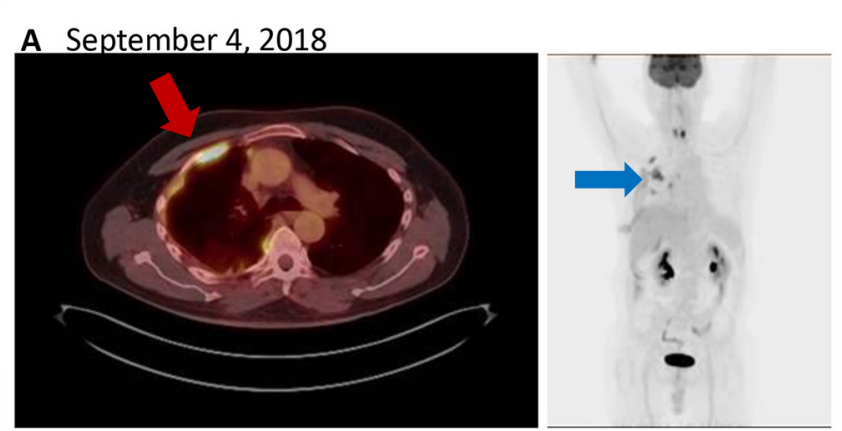

B February 27, 2020

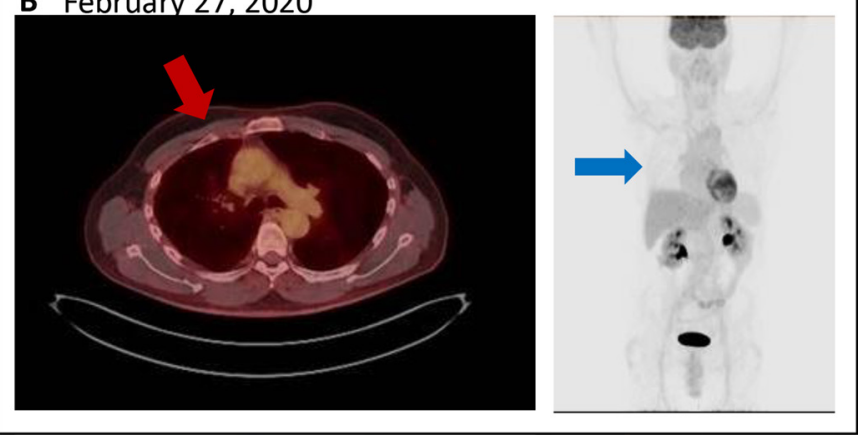

Figure 3 (A) Positron emission tomography/computed tomography (PET/CT) (left) demonstrates hypermetabolic lesion in the pleura of the right anterior rib space (red arrow). Maximum intensity projection (MIP, right) with multifocal lesions (blue arrow). (B) Follow-up PET/CT (left) and MIP (right) with complete metabolic response.

230-430 nm) and extensive podocyte foot process effacement (figure 1F). Due to concern for progressive decline in his kidney function, consideration of immunosuppressive treatment options was discussed with his primary oncologist. The patient was subsequently initiated on rituximab, a monoclonal chimeric antibody binding specifically to CD20 on B lymphocytes with hope that this would not affect any antitumor benefits attributable to $\mathrm{T}$ cell immunity. Nivolumab was held while two infusions of rituximab at $1 \mathrm{~g}$ were administered 14 days apart. Lab results 1 month after rituximab treatment showed an improved serum creatinine of $1.01 \mathrm{mg} / \mathrm{dL}$, decrease in proteinuria by over $50 \%$ ( $4.6 \mathrm{~g} /$ day $)$, and albumin level of $2.9 \mathrm{~g} / \mathrm{dL}$. Flow cytometry analysis of CD19+ B cell counts confirmed complete depletion and undetectable serum PLA2R level (baseline level unavailable). The patient continued to receive nivolumab and has since received five cycles of nivolumab following rituximab therapy with stable to improved kidney function (figure 2). PET/CT imaging continues to show complete metabolic response and no new sites of increased fluorodeoxyglucose activity suspicious for neoplasia (figure 3).

\section{DISCUSSION}

As the clinical indications for immune checkpoint inhibitors has increased in recent years, a greater understanding of the pathogenesis and treatment of those with pre-existing autoimmune disease and cancer represents a major challenge. We report the first case of CPI-associated reactivation of primary $\mathrm{MN}$ in a patient receiving antiPD1 therapy responsive to rituximab.

The pathophysiology of CPI-associated irAEs is not completely understood. CPI increases antitumor immunity by blocking intrinsic down-regulators of immunity. This can lead to the activation of autoreactive $\mathrm{T}$ cells, subsequent production of proinflammatory cytokines, and an autoimmune humoral response. ${ }^{5}$ Current literature suggests that over one-third of patients with pre-existing rheumatic or autoimmune disease have experienced flares of their prior disorder in association with treatment using immune CPIs for malignancy. ${ }^{67}$ Disease flares were more commonly reported with anti-PD-1/PD-L1 agents than with CTLA-4 blockade (62\% vs 36\%), whereas de novo irAEs were more common among patients treated with CTLA- 4 blockade ( $42 \%$ vs $26 \%$ ). ${ }^{6}$ Those with adverse events were more likely to have a partial or complete tumorous response, compared with those who did not have adverse events. ${ }^{6}$ While there appears to be a correlative relationship between development of irAEs and efficacy of treatment, further prospective studies are needed to define the causal relationship and how it will impact clinical decision-making.

Most reports of CPI-associated reactivation of autoimmune disease describe patients with rheumatological features. To our knowledge, this is the first case of reactivated autoimmune MN following CPI therapy responsive to rituximab. $\mathrm{MN}$ is the most common cause of non-diabetic nephrotic syndrome in adults. The term membranous nephropathy reflects the histologic changes noted on light microscopy, specifically GBM thickening. Primary or idiopathic MN account for most MN cases and are accompanied by the appearance of autoantibodies to PLA2R $(70 \%-80 \%$ of primary MN), and a much smaller fraction $(3 \%-5 \%)$ with autoantibodies to thrombospondin type 1 domain containing 7A protein (THSD7A). PLA2R and THSD7A are expressed on glomerular podocytes. ${ }^{18}$ While secondary $\mathrm{MN}$ is associated with hepatitis $\mathrm{B}$ or $\mathrm{C}$, malignancy, and certain drugs (eg, non-steroidal anti-inflammatory, gold, captopril, penicillamine) accounts for $20 \%-25 \%$ of adult cases. Current research suggests that anti-PLA2R can be used to differentiate primary MN from secondary or paraneoplastic associated $\mathrm{MN},{ }^{9}$ but cases of malignancies have been reported in those with positive PLA2R status. ${ }^{1011}$ To date, all reported cases of malignant pleural mesothelioma and MN have been PLA2R negative or unknown. ${ }^{12-14}$ In general, positive immunohistochemical stain for PLA2R confirms the diagnosis of primary $\mathrm{MN}$; in lieu of biopsy positive serological PLA2R levels can also be used, and if detectable, serve as a surrogate to monitor immunologic activity and disease course. ${ }^{15}$ Serologic response frequently precedes the decline in proteinuria due to delay in functional recovery of the glomerular filtration barrier and podocyte remodeling from the immunologic damage.

Nivolumab-associated reactivation of primary $\mathrm{MN}$ was strongly suspected in our patient, but usage of CPIs can 
also be associated with other immune-mediated kidney diseases. These renal pathologies are varied. In addition to acute tubulointerstitial nephritis, other manifestations include lupus nephropathy, thrombotic microangiopathy, focal segmental glomerulosclerosis, minimal-change disease, secondary or PLA2R negative MN, pauci-immune glomerulonephritis, IgA nephropathy, C3 glomerulopathy, and AA amyloidosis. ${ }^{2} 16$ Thus, renal biopsy for definitive diagnosis was imperative to determine etiology and treatment for our patient.

MPM is a rare and often fatal cancer. Those with the sarcomatoid and biphasic histologic subtypes have more aggressive disease. ${ }^{17}$ Our patient experienced a durable response with nivolumab therapy suggesting that activated $\mathrm{T}$ lymphocytes were successfully targeting cancer cells. Unfortunately, this treatment led to the development of Type 1 diabetes and likely reactivated his underlying autoimmune disease. Based on his clinical and immunologic profile including: worsening performance status, severe lower extremity edema, progressive decline in kidney function from active $\mathrm{MN}$, severe nephrotic syndrome (total urine protein $>13 \mathrm{~g} /$ day and serum albumin $<1.9 \mathrm{~g} / \mathrm{dL}$ ), positive PLA2R status, and continued immune activation from CPI therapy, our patient was considered high to very high risk for progressive functional kidney decline.

Current treatment guidelines for $\mathrm{MN}$ recommend immunosuppressive therapy with cyclophosphamide and steroids, rituximab, or cyclosporine. Of these options, all except for rituximab directly or indirectly suppress $\mathrm{T}$ cell function potentially impacting the antitumor effectiveness of CPI therapy. Cyclophosphamide, an alkylating agent, non-discriminatorily crosslinks DNA resulting in absolute lymphopenia in both $\mathrm{T}$ and $\mathrm{B}$ cells and is associated with increase cancer risk. Cyclosporine, a calcineurin inhibitor, blocks transcription of interleukin 2, suppressing T cell activation and, while it is effective in treating proteinuria, has a high rate of relapse following discontinuation of therapy. ${ }^{18}$ Due to its specificity in targeting B-lymphocytes, rituximab seemingly provided the best option to treat our patient's antibody-mediated glomerular disease without directly affecting his cancer therapy. In situations where $\mathrm{T}$ cell sparing agents may not be an option, then competing risks must be considered in managing patients with declining kidney function and treatment of cancer. Fortunately, our patient responded to rituximab with improved kidney function and has continued to receive nivolumab with stable kidney function.

Since B cells likely contribute to CPI-induced reactivation or de novo autoimmune irAEs, ${ }^{19}$ treatment with rituximab has been successful in several cases of CPI-induced irAEs. Majority of these diseases had pre-existing indications or offlabel use for rituximab such as bullous pemphigold, ${ }^{20}$ idiopathic thrombocytopenic purpura, ${ }^{21}$ Sjogren's syndrome, ${ }^{22}$ pauci-immune glomerulonephritis, ${ }^{2}$ and granulomatous necrotizing vasculitis. ${ }^{2}$ Use of rituximab for CPI-related renal vasculitis was further demonstrated by Mamlouk et al. - all cases achieved partial or complete renal recovery following rituximab treatment. ${ }^{23}$ This could suggest that while CPIs may trigger the irAE(s), the underlying B cell pathophysiology of the disease remains unchanged.

Finally, the role of $\mathrm{B}$ cells in cancer prognosis and immune checkpoint therapy is unknown in MPM. In other cancers such as melanoma, the role of B cells in melanoma progression is controversial, as both protumor and antitumor B cell functions have been reported. ${ }^{24}$ Studies of other solid tumors have identified various B cell subsets as critical protumorous mediators of malignancy ${ }^{25}$ if so, these findings would support B cell depletion or Bruton's tyrosine kinase inhibition along with checkpoint inhibition as an appealing strategy ${ }^{26}$ In general, B cell infiltration in mesothelioma is sparse, although a subgroup with higher numbers of $\mathrm{B}$ cells is described. ${ }^{27}$ In mesothelioma epithelioid type, B cell infiltration has been associated with better patient survival, ${ }^{28}$ but this association is not known in the biphasic sarcomatoid type. Understanding the role of $\mathrm{B}$ cells in specific cancer progression could be beneficial for those with pre-existing autoimmune disease and cancer diagnosis, allowing for either complementary or split treatment should irAEs occur. The clinical application of B cell monitoring could lead to earlier irAE intervention, reduced irAE severity, and direct treatment strategy and/or predict clinical outcomes. More research is needed for determining these clinical applications.

In conclusion, we have shown that rituximab was effective in treating CPI-associated reactivation of primary MN. The patient was successfully re-challenged with nivolumab and maintained stable kidney function and sustained clinical anti-tumor effect. Exacerbation of preexisting autoimmune diseases from immune checkpoint blockade is common, and therapy for autoimmune reactivation should be rationally directed by an understanding of the immunosuppressive mechanism in concert with goals of cancer treatment.

Twitter Jamie S Lin @JamieLinMD and Cassian Yee @tcellsrus

Acknowledgements We thank the patient and his family for providing permission to report adverse events and for their participation and contribution to research.

Contributors Data acquisition was performed by JSL, DYW, and AA. WFG performed the histological examination of the kidney biopsies and contributed in writing the pathology section of the manuscript. The manuscript was prepared by JSL and edited by DYW, OM, WFG, MA, CY, and AA. All the authors contributed to the quality control data, analysis, interpretation of data and writing and final proof of paper. All authors read and approved the final manuscript.

Funding The University of Texas MD Anderson Cancer Center is supported in part by the National Institutes of Health through Cancer Center Support Grant P30CA016672. JSL is supported by National Institutes of Health (K08 DK119466). $\mathrm{CY}$ is member of Parker Institute of Cancer Immunotherapy.

Competing interests None declared.

Patient consent for publication Obtained.

Ethics approval This study was approved by the institutional review board in accordance with the principles of the Declaration of Helsinki.

Provenance and peer review Not commissioned; externally peer reviewed.

Open access This is an open access article distributed in accordance with the Creative Commons Attribution Non Commercial (CC BY-NC 4.0) license, which permits others to distribute, remix, adapt, build upon this work non-commercially, and license their derivative works on different terms, provided the original work is properly cited, appropriate credit is given, any changes made indicated, and the use is non-commercial. See http://creativecommons.org/licenses/by-nc/4.0/. 


\section{ORCID iDs}

Jamie S Lin http://orcid.org/0000-0002-7073-9658

Omar Mamlouk http://orcid.org/0000-0002-1242-2740

\section{REFERENCES}

1 Beck LH, Bonegio RGB, Lambeau G, et al. M-type phospholipase A2 receptor as target antigen in idiopathic membranous nephropathy. $N$ Engl J Med 2009;361:11-21.

2 Mamlouk O, Selamet U, Machado S, et al. Nephrotoxicity of immune checkpoint inhibitors beyond tubulointerstitial nephritis: single-center experience. j. immunotherapy cancer 2019;7:2.

3 Lefaucheur C, Stengel B, Nochy D, et al. Membranous nephropathy and cancer: epidemiologic evidence and determinants of high-risk cancer association. Kidney Int 2006;70:1510-7.

4 Plaisier E, Ronco P. Screening for cancer in patients with glomerular diseases. Clin J Am Soc Nephrol 2020;15:886-8.

5 Tahir SA, Gao J, Miura Y, et al. Autoimmune antibodies correlate with immune checkpoint therapy-induced toxicities. Proc Natl Acad Sci U $S$ A 2019;116:22246-51.

6 Abdel-Wahab N, Shah M, Lopez-Olivo MA, et al. Use of immune checkpoint inhibitors in the treatment of patients with cancer and preexisting autoimmune disease: a systematic review. Ann Intern Med 2018;168:121-30.

7 Johnson DB, Beckermann KE, Wang DY. Immune checkpoint inhibitor therapy in patients with autoimmune disease. Oncology 2018;32:190-4.

8 Tomas NM, Beck LH, Meyer-Schwesinger C, et al. Thrombospondin type-1 domain-containing $7 \mathrm{~A}$ in idiopathic membranous nephropathy. N Engl J Med 2014;371:2277-87.

9 Timmermans SAMEG, Ayalon R, van Paassen P, et al. Antiphospholipase A2 receptor antibodies and malignancy in membranous nephropathy. Am J Kidney Dis 2013;62:1223-5.

10 Bjørneklett R, Vikse BE, Svarstad E, et al. Long-term risk of cancer in membranous nephropathy patients. Am J Kidney Dis 2007;50:396-403.

$11 \mathrm{Kim}$ Y. Relapse of membranous nephropathy with cancer immunotherapy. Clin Kidney J 2019;33

12 Sakamoto K, Suzuki H, Jojima T. Membranous glomerulonephritis associated with diffuse malignant pleural mesothelioma: report of a case. Surg Today 2000;30:1124-6.

13 Pu X, Dou Y, Liu D, et al. Membranous nephropathy associated with malignant pleural mesothelioma in an adult patient: a case report. Mol Clin Oncol 2016;5:407-10.
14 Galesic K, Bozic B, Heinzl R, et al. Pleural mesothelioma and membranous nephropathy. Nephron 2000;84:71-4.

15 Hofstra JM, Beck LH, Beck DM, et al. Anti-phospholipase $\mathrm{A}_{2}$ receptor antibodies correlate with clinical status in idiopathic membranous nephropathy. Clin J Am Soc Nephrol 2011;6:1286-91.

16 Cortazar FB, Marrone KA, Troxell ML, et al. Clinicopathological features of acute kidney injury associated with immune checkpoint inhibitors. Kidney Int 2016:90:638-47.

17 Tsao AS, Wistuba I, Roth JA, et al. Malignant pleural mesothelioma. J Clin Oncol 2009;27:2081-90.

18 Fervenza FC, Appel GB, Barbour SJ, et al. Rituximab or cyclosporine in the treatment of membranous nephropathy. $N$ Engl J Med 2019;381:36-46.

19 Das R, Bar N, Ferreira M, et al. Early B cell changes predict autoimmunity following combination immune checkpoint blockade J Clin Invest 2018;128:715-20.

20 Sowerby L, Dewan AK, Granter S, et al. Rituximab treatment of Nivolumab-Induced bullous pemphigoid. JAMA Dermatol 2017:153:603-5.

21 Shiuan E, Beckermann KE, Ozgun A, et al. Thrombocytopenia in patients with melanoma receiving immune checkpoint inhibitor therapy. J Immunother Cancer 2017;5:8.

22 Ghosn J, Vicino A, Michielin O, et al. A severe case of neuroSjögren's syndrome induced by pembrolizumab. J Immunother Cancer 2018;6:110.

23 Mamlouk O, Lin JS, Abdelrahim M, et al. Checkpoint inhibitor-related renal vasculitis and use of rituximab. Journal for ImmunoTherapy of Cancer 2020;8:e000750.

24 Griss J, Bauer W, Wagner C, et al. B cells sustain inflammation and predict response to immune checkpoint blockade in human melanoma. Nat Commun 2019;10:4186.

25 Petitprez F, de Reyniès A, Keung EZ, et al. B cells are associated with survival and immunotherapy response in sarcoma. Nature 2020;577:556-60.

26 Somasundaram R, Zhang G, Fukunaga-Kalabis M, et al. Tumorassociated B-cells induce tumor heterogeneity and therapy resistance. Nat Commun 2017;8:607.

27 Minnema-Luiting J, Vroman $\mathrm{H}$, Aerts J, et al. Heterogeneity in immune cell content in malignant pleural mesothelioma. Int $\mathrm{J} \mathrm{Mol} \mathrm{Sci}$ 2018;19. doi:10.3390/ijms19041041. [Epub ahead of print: 30 Mar 2018].

28 Chee SJ, Lopez M, Mellows T, et al. Evaluating the effect of immune cells on the outcome of patients with mesothelioma. $\mathrm{Br} J$ Cancer $2017 ; 117: 1341-8$ 